\title{
Evidências Neurobiológicas de Viés Atencional no Transtorno Obsessivo-Compulsivo: Revisão Sistemática
}

\author{
Samara dos Santos Ribeiro* (•, Paulo Roberto Cabral Passos (®), \\ \& Marcele Regine de Carvalho (1) \\ Universidade Federal do Rio de Janeiro, Rio de Janeiro, RJ, Brasil
}

\begin{abstract}
RESUMO - O viés atencional corresponde à alocação de recursos de atenção a materiais irrelevantes à tarefa. Supõe-se que pacientes com transtorno obsessivo-compulsivo (TOC) apresentem viés atencional voltado à ameaça. Com o objetivo de descrever os achados neurobiológicos do viés atencional voltado à ameaça no TOC, foi realizada uma busca sistemática por estudos experimentais com investigação neurobiológica nas bases de dados: MEDLINE, Web of Science, Scopus e LILACS. Quatro estudos com grupo controle são descritos nos resultados, todos indicam diferenças estatisticamente significativas na atividade encefálica associada a atenção em pacientes. Os achados neurobiológicos dos estudos incluídos na revisão sugerem a alocação de recursos da atenção a estímulos irrelevantes, independente da valência emocional no TOC.
\end{abstract}

PALAVRAS-CHAVE: transtorno obsessivo-compulsivo, viés atencional, neurobiologia

\section{Neurobiological Evidence of Attentional Bias in Obsessive-Compulsive Disorder: Systematic Review}

\begin{abstract}
The attentional bias is the allocation of attentional resources to irrelevant stimuli during a task. It is assumed that patients with obsessive-compulsive disorder (OCD) presents attentional bias toward threatening stimuli. In order to describe OCD's neurobiological findings of attentional bias towards a threat, it was performed a systematic review of experimental studies with neurobiological assessment in the following databases: MEDLINE, Web of Science, Scopus and LILACS. Four studies with control group are described in this review, all presenting statistically significant differences in brain activity associated with attention in patients. The neurobiological findings of the studies included in the review suggest allocation of attentional resources to irrelevant stimuli, regardless of the emotional valence in OCD.
\end{abstract}

KEYWORDS: obsessive-compulsive disorder, attention, neurobiology

\section{INTRODUÇÃO}

O transtorno obsessivo-compulsivo (TOC) é caracterizado por obsessões, que são pensamentos, imagens e impulsos intrusivos, indesejáveis e recorrentes; e/ou compulsões, que são rituais comportamentais ou mentais repetitivos, realizados para reduzir o sofrimento causado pela obsessão, ou realizados conforme regras rígidas determinadas pelo indivíduo (American Psychiatric Association [APA], 2013). Estudos em pacientes com TOC tem testado hipóteses de inadequações no processamento da informação nos sistemas de memória e atenção, como exemplo, o viés atencional voltado à ameaça (Muller \& Roberts, 2005).

O TOC é um transtorno com fisiopatologia ainda desconhecida, o modelo teórico, mais citado atualmente tem forte apoio empírico e envolve alterações no circuito córtico-estriado-tálamo-cortical (CSTC). Sugere-se um desequilíbrio entre o circuito direto (excitatório): córtex orbitofrontal (OFC)-estriado-globo pálido-tálamo-córtex e o indireto (inibitório): córtex pré-frontal dorsolateral

* E-mail: sam.s.ribeiro@gmail.com

- Submetido: 06/07/2016; Revisado: 11/11/2017; Aceito: 04/03/2018. 
(DLPFC)-estriado-globo pálido-núcleo subtalâmico-córtex. Alterações nestes circuitos e na interação entre eles, como a hiperativação OFC-estriado e OFC-tálamo podem causar os sintomas observados no TOC. Ademais, existem áreas envolvidas nesses circuitos que aparecem frequentemente alteradas em estudos com recursos de imagem em pacientes com TOC, como núcleo caudado, córtex cingulado anterior (ACC), gânglio basal e tálamo (Kwon et al., 2009; Nakao et al., 2014; Rauch, 2003).

Referente à atenção, estudos em humanos e animais promoveram uma série de informações sobre este sistema, no entanto, seus mecanismos e redes neuronais não estão claros (Petersen \& Posner, 2012). Atualmente, considera-se que o sistema da atenção é composto por três subsistemas: alerta, orientação e controle executivo. Cada subsistema possui mecanismos, funções e vias anatômicas distintas. Supõe-se que o estado de alerta seja iniciado com a atividade do locus cæruleus ou cerúleo, no tronco cerebral, que recebe aferência do sistema sensorial e projeta para o córtex frontal e parietal direitos. $\mathrm{O}$ estado de alerta ainda pode envolver áreas como o tálamo, cíngulo anterior e adjacências (Lima, 2005; Petersen \& Posner, 2012; Posner, 1992).

A orientação da atenção a determinado material pode ser possível através de duas redes neuronais: dorsal, com início da atividade do campo visual frontal e envolvendo as regiões do sulco intraparietal e lobo parietal superior; ventral com atividade de regiões da junção temporo-parietal e do córtex frontal ventral. O prosencéfalo e lobo parietal superior também são associados à orientação em alguns estudos (Petersen \& Posner, 2012). O controle executivo é, possivelmente, o subsistema que determina se a atenção deve ser mantida em determinado material, de acordo com a relevância atribuída, e inibe a atenção a outros materiais. Sugere-se duas redes de processamento distintas: frontoparietal e cingulo-opercular, além do envolvimento de áreas do lobo frontal e córtex pré-frontal (Lima, 2005; Petersen \& Posner, 2012; Posner, 1994).

$\mathrm{O}$ viés atencional voltado à ameaça representa a atenção e associação seletivas, e tendência de acesso a estímulos diretamente relacionados aos medos, angústias e preocupações dos indivíduos. Estímulos irrelevantes ou ambíguos são classificados como altamente ameaçadores por indivíduos ansiosos, se possuírem relação mínima com a causa da sua ansiedade (Beard, 2011; Coles \& Heimberg, 2002; Coles et al., 2007). Uma meta-análise, composta por 172 estudos de investigação de viés atencional, demonstrou que indivíduos ansiosos apresentam viés atencional a estímulos ameaçadores em todos os paradigmas (emotional stroop task, dot-probe e emotional spatial cuing), tipos de estímulos (palavras e imagens) e tempos de exposição (subliminar e supralimar) quando comparados a controles não ansiosos (Bar-Haim et al., 2007).

Existem três componentes de viés atencional voltado à ameaça: facilidade de atenção, dificuldade de desviar a atenção e evitação da atenção (Cisler \& Koster, 2010). A amígdala pode estar envolvida com facilidade de atenção à ameaça, devido ao seu papel no processamento de informações de medo e ameaça (Hartley \& Phelps, 2010). A dificuldade de desviar a atenção pode envolver os córtices pré-frontal, ACC e o OFC, que são geralmente associados ao processamento normal de manutenção e desvio da atenção (Kondo et al., 2004; Wu et al., 2017). Estas áreas exerceriam um controle top-down de estruturas límbicas envolvidas na regulação da emoção e medo (Etkin et al., 2011). A atividade do córtex pré-frontal está inversamente relacionada a maior ou menor distração a ameaça (Bishop et al., 2004; Cisler \& Koster, 2010).

Pacientes com TOC apresentaram viés atencional voltado à ameaça identificado por meio do paradigma stroop, quando comparados a controles saudáveis (Lavy et al., 1994; Rao, et al., 2010). Entretanto, alguns estudos não encontraram diferenças significativas entre pacientes e controles, em tarefas para avaliar a atenção, contradizendo a existência de viés atencional voltado à ameaça (Harkness et al., 2009; Kyrios \& Iob, 1998; Moritz et al., 2008). Atualmente se enuncia que os traços de ansiedade, a valência emocional dos estímulos, a duração deles, comorbidades, idade, tratamento do transtorno, dentre outras variáveis, podem influenciar no viés atencional, seu estágio de processamento (automático ou estratégico) e seu mecanismo (Cisler \& Koster, 2010; Beard, 2011; Mathewset al., 1997; Muller \& Roberts, 2005).

A suspeita de que o viés atencional possa influenciar na fisiopatologia e manutenção do TOC gerou uma quantidade significativa de estudos experimentais, com diferentes paradigmas para avaliação da atenção (para revisão ver Muller \& Roberts, 2005). Contudo, nota-se uma escassez de evidências neurobiológicas da existência do viés em pacientes com TOC uma vez que, a maioria dos estudos seguem procedimentos pautados em tarefas de avaliação da atenção e escalas psicométricas. A proposta desta revisão sistemática foi buscar estudos que investigassem evidências biológicas da existência de viés atencional à ameaça em pacientes com TOC, por meio da avaliação da função cerebral durante tarefas que exigem a ativação do sistema da atenção. $O$ objetivo foi relatar e relacionar as evidências a fim de descrever o cenário atual desta investigação, identificando se a hipótese da existência de viés atencional voltado a materiais ameaçadores pode ser corroborada biologicamente, além de incentivar a produção científica para busca de mais evidências. 


\section{MÉTODO}

Uma busca sistemática foi conduzida, durante todo o mês de fevereiro de 2016, sendo a última busca realizada no dia 04 de março de 2016, nas bases de dados MEDLINE, LILACS, Web of Science, Scopus; estas duas últimas acessadas por meio do portal periódicos CAPES. Os termos para busca foram igualmente aplicados em todas as bases da seguinte forma: attentional bias and obsessive-compulsive disorder and neuroimage, attentional bias and OCD and neuroimage, attentional bias and obsessive-compulsive disorder and neurobiolog, attentional bias and OCD and neurobiolog, attentional bias and obsessive-compulsive disorder e attentinoal bias and OCD. Na base MEDLINE a busca foi realizada em todos os campos; na base LILACS no campo: título, resumo, assunto; na Web of Science, no campo: tópico; e na base Scopus no campo: article, title, abstract, keywords. Os artigos deveriam estar nos idiomas inglês e português, não foram aplicadas delimitações de data e estado de publicação (publicados, aceitos, impressos ou somente on-line).

Os critérios para inclusão nesta revisão foram estudos experimentais com evidências neurobiológicas da existência de viés atencional em pacientes com TOC comparados a controles saudáveis. Os estudos deveriam conter amostras de pacientes diagnosticados com TOC segundo os critérios do Manual Diagnóstico e Estatístico de Transtornos Mentais (DSM, do inglês Diagnostic and Statistical Manual of Mental
Disorders), independente de gênero, idade, comorbidades e tratamento. Não foram determinadas características com relação à severidade e sintomas do TOC ou quaisquer outros aspectos do transtorno. Inicialmente considerou-se somente estudos que utilizassem recursos de imagem, porém, devido a pouca quantidade de artigos encontrados, foram considerados quaisquer estudos independente do instrumento de avaliação neurobiológica utilizado. Artigos de revisão e sobre outros espectros obsessivo-compulsivos foram excluídos.

Os resultados das seis combinações de palavras-chave foram contabilizados e os artigos em duplicata foram removidos. Os estudos encontrados foram avaliados, inicialmente pelo título, sendo descartados aqueles que notoriamente, segundo o avaliador, não seguiam os critérios de inclusão. Após esse processo os artigos foram avaliados por meio da leitura do resumo. Os dados considerados de cada estudo foram o $n$ da amostra, método de diagnóstico, idade média, sexo, lateralidade, uso de medicamentos, metodologia (escalas psicométricas utilizadas, paradigma para avaliação da atenção, método de avaliação neurobiológica, como eletroencefalograma (EEG) e ressonância magnética funcional (RMF), resultados e considerações dos autores. Os dados extraídos foram relatados, comparados e integrados nas sessões seguintes desta revisão.

\section{RESULTADOS}

O total de 209 artigos foi encontrado na busca pelos termos já descritos. Após a remoção de duplicatas (artigos encontrados em mais de uma base), 138 foram analisados pelo título. A avaliação dos títulos excluiu 112 artigos, os quais eram revisões, artigos em idiomas diferentes do inglês e português, e artigos não referentes ao TOC ou que não condiziam com o objetivo desta revisão. Os resumos de 26 artigos foram lidos e somente 4 foram selecionados para avaliação do texto completo e extração de dados. A Figura 1 apresenta o processo de inclusão dos estudos em um fluxograma.

Os estudos selecionados possuíam desenhos semelhantes que incluíam investigação cognitiva de viés atencional e avaliação neurobiológica. Todos os pacientes foram diagnosticados com base na DSM-IV (APA, 1994) e todos os participantes, incluindo controles saudáveis, eram destros. Os estudos estão descritos a seguir e a Tabela 1 resume suas principais características.

Van den Heuvel et al. (2005) comparou 18 pacientes com TOC a 19 controles saudáveis, além de 15 pacientes com Transtorno de Pânico (TP) e 14 com Hipocondria (H). A idade média da amostra do grupo com TOC era de 33,4 anos e a maioria (12) eram mulheres. Os pacientes deveriam suspender a medicação por no mínimo 4 semanas antecedentes ao procedimento. Os questionários utilizados para avaliação dos sintomas nos pacientes com TOC foram: Y-BOCS (Goodman et al., 1989), Padua Inventory (Sanavio, 1988) e Whiteley Index (Pilowsky, 1967). Para a investigação de viés antencional utilizou-se o paradigma stroop emocional (Jensen \& Rohwer, 1966), uma das mais utilizadas na literatura em indivíduos ansiosos (van den Heuvel et al., 2005). Na tarefa stroop o indivíduo deve nomear a cor em que uma palavra está impressa, ignorando a sua grafia (Jensen \& Rohwer Jr., 1966). Na versão stroop emocional são inseridos estímulos ameaçadores para a população a ser avaliada, os quais, teoricamente, deveriam provocar reações distintas das provocadas por estímulos neutros, geralmente aumentando o tempo de reação. Contudo, alguns autores contrapõem esta hipótese atribuindo os elevados tempos de reação a um processamento tardio não relacionado à atenção, como o estado afetivo negativo em indivíduos ansiosos (Bar-Haim et al., 2007).

Na versão do referido estudo, além das condições padrão, congruentes (palavras e cor iguais, vermelho impresso em vermelho) e incongruentes (palavra e cor diferentes, vermelho impresso na cor azul), a tarefa incluiu condições 
Tabela 1

Resumo da metodologia, resultados e conclusões dos artigos selecionados

\begin{tabular}{|c|c|c|c|c|c|c|}
\hline Autor & $\mathbf{N}$ & $\begin{array}{l}\text { Avaliação } \\
\text { cognitiva }\end{array}$ & $\mathbf{A B}^{1}$ & Medicação & Resultados (TOC vs controles) & Conclusão \\
\hline $\begin{array}{l}\text { van den } \\
\text { Heuvel et } \\
\text { al.,2005 }\end{array}$ & 18 & $\begin{array}{l}\text { Stroop } \\
\text { emocional }\end{array}$ & RMF & $\begin{array}{l}\text { sem medicação por } \\
4 \text { semanas }\end{array}$ & $\begin{array}{l}\text { TR para condições incongruentes } \\
\text { marginalmente maior; Associação } \\
\text { entre a escala de sofrimento e aumento } \\
\text { do TR em palavras do TOC; Taxa } \\
\text { de erro maior; Maior ativação do } \\
\text { precuneus direito, giro parahipocampal } \\
\text { esquerdo e tronco cerebral esquerdo } \\
\text { em incongruentes vs congruentes; } \\
\text { Maior ativação da amígdala bilateral e } \\
\text { hipotálamo esquerdo em palavras TOC }\end{array}$ & $\begin{array}{l}\text { Atenção a estímulos } \\
\text { irrelevantes associada a } \\
\text { áreas fronto-estriatais, } \\
\text { límbicas e ventrais, sem } \\
\text { indicação de viés pelo } \\
\text { Stroop em pacientes. }\end{array}$ \\
\hline $\begin{array}{l}\text { Ischebeck } \\
\text { et al., } \\
2011\end{array}$ & 20 & $\begin{array}{l}\text { tarefa de } \\
\text { reconhecimento } \\
\text { visual + sons }\end{array}$ & EEG & $\begin{array}{l}\text { metade livre de } \\
\text { medicação }\end{array}$ & $\begin{array}{l}\text { Aumento da amplitude novelty } \mathrm{P} 3 \text { em } \\
\text { regiões centrais, frontais e parietais } \\
\text { do hemisfério esquerdo em condições } \\
\text { negativas; Aumento da amplitude } \\
\text { novelty P3 em regiões centrais e } \\
\text { frontais no hemisfério esquerdo em } \\
\text { condições neutras }\end{array}$ & $\begin{array}{l}\text { Resposta orientada a } \\
\text { estímulos inesperados } \\
\text { supostamente aumentada } \\
\text { independente da } \\
\text { valência emocional. } \\
\text { Hipersensibilidade do } \\
\text { sistema atencional a } \\
\text { estímulos salientes. }\end{array}$ \\
\hline $\begin{array}{l}\text { Thomas } \\
\text { et al., } \\
2013\end{array}$ & 20 & $\begin{array}{c}\text { Stroop } \\
\text { emocional }\end{array}$ & EEG & $\begin{array}{l}\text { sem uso de } \\
\text { benzodiazepínicos } \\
\text { por } 48 \mathrm{~h}\end{array}$ & $\begin{array}{l}\text { Maior TR no stroop no geral e maior } \\
\text { TR para palavras de ameaça vs neutras; } \\
\text { Aumento de amplitude P1 para ameaça } \\
\text { vs neutra; Topografia atipica de P1, } \\
\text { redução fronto-parietal comparada a } \\
\text { região central; Aumento da latência de } \\
\text { N1 para ameaça vs neutra; Grupo com } \\
\text { interferência do efeito stroop (10 com } \\
\text { TOC) teve aumento na amplitude P1 e } \\
\text { P3 durante estímulos ameaçadores }\end{array}$ & $\begin{array}{l}\text { Viés atencional precoce } \\
\text { voltado à ameaça. }\end{array}$ \\
\hline $\begin{array}{l}\text { Fan } e t \\
\text { al., } 2014\end{array}$ & 31 & $\begin{array}{c}\text { Stroop } \\
\text { emocional }\end{array}$ & EEG & $\begin{array}{l}\text { livres de } \\
\text { medicação }\end{array}$ & $\begin{array}{l}\text { TR maiores; Níveis positivos e } \\
\text { negativos de interferência do stroop } \\
\text { similares; Maior amplitude P2 em } \\
\text { regiões frontais e centrais comparadas } \\
\text { a parietais; Maior amplitude P } 3 \text { para } \\
\text { palavras negativas vs neutras }\end{array}$ & $\begin{array}{l}\text { O TOC pode ser } \\
\text { caracterizado por uma } \\
\text { disfunção da inibição da } \\
\text { atenção para informações } \\
\text { irrelevantes. }\end{array}$ \\
\hline
\end{tabular}

Nota. AB: avaliação biológica; TR: tempo de reação, RMF: Ressonância Magnética Funcional; EEG: Eletroencefalograma.

com palavras de valência emocional negativa relacionadas ao TOC, TP e hipocondria $(\mathrm{H})$ e palavras neutras (van den Heuvel et al., 2005). O recurso utilizado na avaliação neurobiológica foi RMF, com dados coletados durante a realização da tarefa. A apresentação dos estímulos foi supraliminar com duração de 2 segundos. Os estímulos supraliminares são percebidos conscientemente, o que não ocorre com os subliminares, que são apresentados por no máximo $50 \mathrm{~ms}$, e são processados inconscientemente. Ao final da tarefa, os participantes deveriam responder a uma escala de avaliação de sofrimento provocado pelas palavras (van den Heuvel et al., 2005).

Os resultados do efeito stroop emocional para pacientes com TOC foram os seguintes: tempo de reação (TR) marginalmente maior para condições incongruentes comparados a controles; associação significativa entre os escores da escala de sofrimento e TR maiores para palavras relacionadas ao TOC; taxas de erro maiores que no grupo controle. Com estes resultados os autores concluíram que pacientes com TOC apresentaram maior interferência cognitiva, apesar de não apresentarem viés atencional voltado à ameaça quando comparados a controles saudáveis. Com relação aos achados neurobiológicos, o grupo TOC apresentou ativação específica de regiões posteriores do encéfalo. Em resposta a condições incongruentes da tarefa foi observado aumento da ativação no precuneus direito, giro parahipocampal esquerdo e tronco cerebral esquerdo no grupo de pacientes com TOC comparado ao grupo controle (van den Heuvel et al., 2005).

Durante a apresentação de palavras relacionadas ao TOC, toda a amostra de pacientes (i.e., TOC, TP e H) apresentou maior ativação da região fronto-estriatal. Os pacientes com TOC, especificamente, tiveram um aumento da ativação da amígdala bilateral e do hipotálamo esquerdo quando comparados a controles. Uma análise de correlação mostrou que o estado de ansiedade no TOC está relacionado com o aumento da ativação do córtex pré-frontal ventrolateral (VLPFC) esquerdo e córtex extra-estriado esquerdo, quando comparadas a apresentação de palavras relacionadas aos sintomas e neutras (van den Heuvel et al., 2005). Segundo 


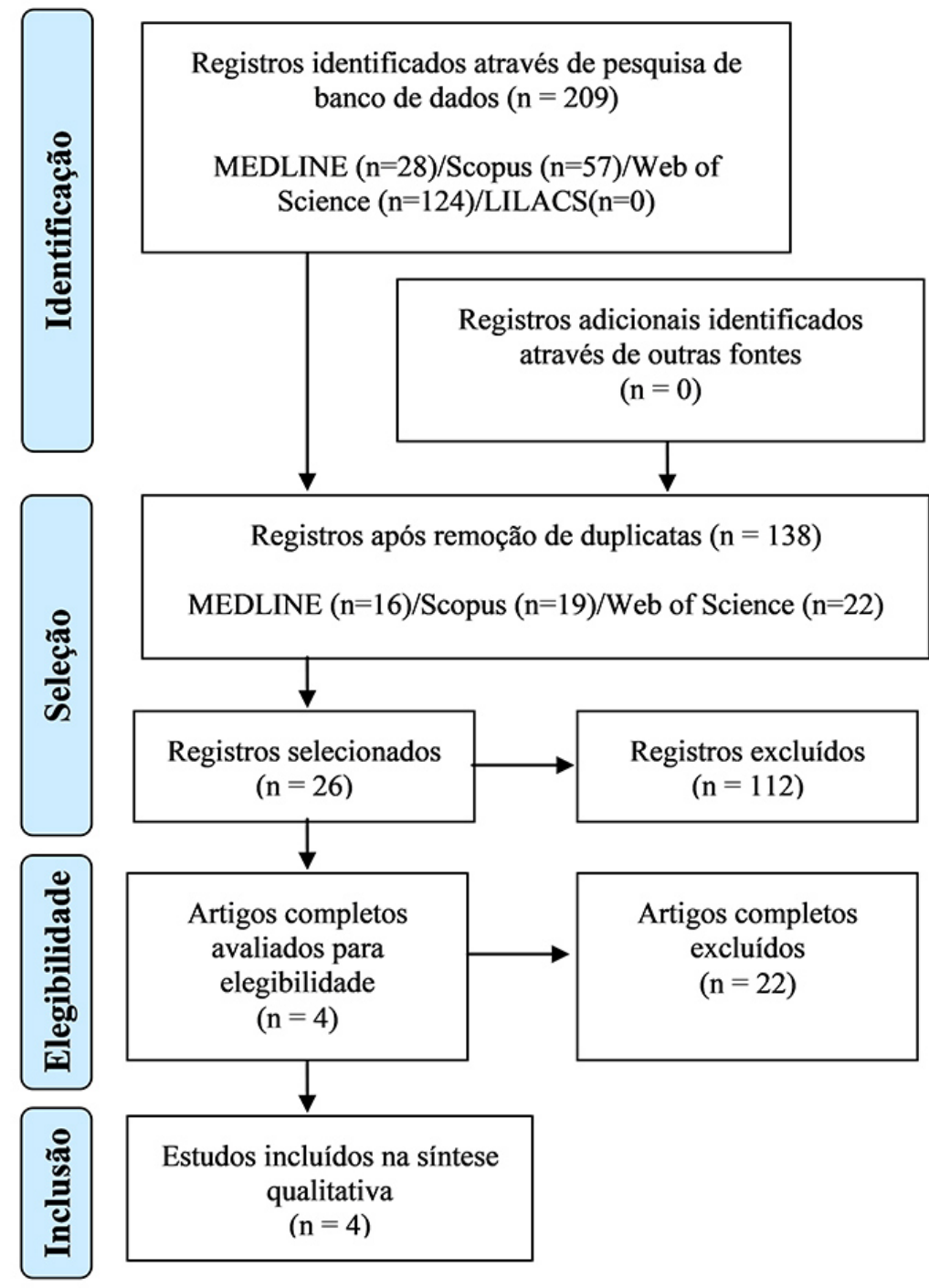

Figura 1. Processo de seleção dos estudos por meio da identificação, seleção, elegibilidade e inclusão, de acordo com as recomendações do PRISMA.

os autores, os resultados apontam para o processamento automático de estímulos emocionais relacionados ao TOC, pelo qual o sistema ventral é responsável (van den Heuvel et al., 2005).

Os próximos estudos relatados utilizaram o EEG para avaliação neurobiológica. O Potencial Relacionado a Evento (ERP) é uma alteração elétrica cortical detectável após exposição à algum estímulo. Os componentes do ERP são ondas identificadas de acordo com a polaridade do pico de amplitude alcançado. Assim, os picos descritos a seguir como P1, P2 e P3 referem-se a um pico de amplitude positivo (P) alcançado após 100, 200 e $300 \mathrm{~ms}$, respectivamente, após o início da exposição a um estímulo (evento). O pico N1, possui polaridade negativa e também ocorre $100 \mathrm{~ms}$ após o início do evento (Key et al., 2005).

$\mathrm{O}$ pico P1 está sempre presente em exposições a estímulos visuais, pode estar relacionado à atenção sensorial e aos níveis de excitação. A amplitude é maior sobre regiões occipitais e é variável de acordo com a atenção atribuída (Vogel \& Luck, 2000). O pico P2 é identificado em áreas frontais e está relacionado à modulação da atenção a estímulos não-alvo e à classificação de estímulos (Key et al., 2005). A presença e amplitude do pico P3 parecem estar relacionadas à atenção, probabilidade de exposição a um estímulo, estímulos relevantes, quantidade de recursos de processamento disponíveis, qualidade e alocação da atenção (Friedman et al., 2001). Por sua vez, A novelty P3 é um dos componentes de P3 detectada após eventos inesperados, especialmente sons, durante uma tarefa. Em regiões posteriores do encéfalo a novelty $P 3$ permanece estável na presença e ausência de novos estímulos, e é maior após eventos recorrentes podendo, portanto, estar relacionada à categorização (Friedman et al., 2001). O pico $\mathrm{N} 1$ pode refletir a atenção seletiva às características básicas 
dos estímulos, início do reconhecimento de padrões tardios e processamento de discriminação intencional (Vogel \& Luck, 2000).

O estudo de Ischebeck et al. (2011) contou com 20 pacientes com TOC, a maioria do sexo feminino (13) e metade faziam uso de medicamentos para o TOC. O grupo controle era composto por 20 indivíduos saudáveis. Os questionários de avaliação dos sintomas e da ansiedade foram MADRS (Montgomery \& Asberg, 1979), BDI (Beck et al., 1961), Y-BOCS, OCI (Foa et al., 1998) e STAI (Spielberger et al., 1983). O teste cognitivo para avaliação da atenção foi uma tarefa de reconhecimento visual, com blocos intercalados com 600 sons, dos quais 120 eram nãorepetitivos e complexos e 480 eram padrão, cada um com duração de $200 \mathrm{~ms}$. A tarefa de reconhecimento visual era composta por 2 condições: neutra (imagens sem valência emocional) e negativa (imagens com valência emocional negativa e neutras). Não havia imagens relacionadas diretamente aos sintomas do TOC. As imagens eram antecedidas pelos sons e expostas por 1 segundo, os indivíduos deveriam responder se eram novas ou repetidas. Ao final da tarefa, os participantes deveriam classificar as imagens em excitantes ou indesejáveis, e o desejo de realizar as compulsões provocadas por elas (Ischebeck et al., 2011).

$\mathrm{O}$ estudo avaliou o componente de ERP novelty P3. Nos pacientes as condições negativas da tarefa provocaram aumento na amplitude dos sinais novelty $\mathrm{P} 3$ na região frontocentral (vértex) e central, frontal, parietal, intersecções centro-frontal e centro-parietal do hemisfério esquerdo, comparados aos controles. Condições neutras também provocaram aumento da amplitude na região fronto-central (vértex) e frontal, intersecção fronto-central do hemisfério esquerdo, quando comparados aos controles (Ischebeck $e t$ al., 2011).

Não houve diferenças entre os grupos com relação à latência de novelty $P 3$ e nenhuma correlação entre as amplitudes e os escores dos questionários. As taxas de provocação dos sintomas foram altas para imagens negativas. Segundo os autores, os resultados demonstraram diferenças da novelty $P 3 \mathrm{em}$ áreas frontais, centrais e parietais do hemisfério esquerdo e sugerem que esta é reforçada em pacientes com TOC. Esta característica da novelty $P 3$ pode refletir uma melhor resposta a estímulos inesperados independente da valência emocional (Ischebeck et al., 2011).

Thomas et al. (2013) em seu estudo incluíram um total de 60 participantes, dentre os quais 20 tinham TOC, 20 TP com ou sem agorafobia e 20 controles saudáveis. Os pacientes deveriam interromper o uso de benzodiazepínicos 48 horas antes do procedimento. Algumas escalas para avaliação dos sintomas foram aplicadas como BSI (Derogatis \& Melisaratos, 1983), Padua Inventory e Y-BOCS. A versão emocional do stroop foi novamente utilizada com palavras relacionadas aos sintomas do TOC personalizadas para cada paciente. As peculiaridades desta versão específica do stroop emocional foram a utilização de 3 condições: palavras relacionadas ao TOC, palavras relacionadas ao pânico e palavras neutras. Estas palavras foram selecionadas antes do procedimento, de acordo com a angústia que provocavam, medida por uma escala (pontuação de 0 a 4). As 10 palavras mais e menos angustiantes formavam os grupos muito e pouco estimulantes, respectivamente, de cada participante (Thomas et al., 2013).

Desta forma a tarefa foi organizada em 4 blocos, metade com palavras neutras e muito estimulantes e metade com palavras neutras e pouco estimulantes. A duração de cada estímulo foi de $200 \mathrm{~ms}$ e a tarefa foi aplicada durante captação de EEG. Em geral, pacientes (i.e. TOC e TP) tiveram maior TR que controles. Pacientes com TOC apresentaram maior amplitude de P1 durante estímulos ameaçadores, comparados a pacientes com TP e controles. A latência de N1, durante estímulos ameaçadores comparados a neutros foi também maior no TOC, que nos outros grupos. Uma redução da amplitude $\mathrm{P} 1$ em regiões fronto-parietais relacionadas a regiões centrais foi encontrada nas duas amostras clínicas (Thomas et al., 2013).

Análises complementares foram feitas com base no escore de interferência da tarefa stroop emocional. O escore de interferência foi obtido por meio da subtração da média do TR para palavras neutras da média do TR para palavras pessoalmente ameaçadoras. A partir deste ponto houve a divisão em 2 grupos, o de interferência $(n=38)$ e o de não-interferência $(n=22), 10$ dos 20 pacientes com TOC foram alocados no grupo interferência. Os resultados para o primeiro grupo foram: maior TR geral, e maior TR para condições de ameaça comparadas a neutras. As amplitudes P1 e P3 foram maiores para estímulos ameaçadores que neutros no grupo de interferência, sendo que no grupo não-interferência o comportamento de $\mathrm{P} 3$ foi o oposto, ou seja, menor amplitude deste componente para ameaças comparadas a estímulos neutros (Thomas et al., 2013).

A conclusão dos autores foi de que há existência de viés atencional precoce voltado a ameaça em pacientes com TOC. Os resultados do EEG podem indicar aumento da atenção sensorial em relação à ameaça, processamento precoce da ameaça reforçado e mais complexo e prolongado durante estágio de discriminação de estímulo (Thomas et al., 2013).

Fan et al. (2014) utilizaram uma amostra com TOC e a compararam a controles saudáveis. Foram 31 pacientes, destes 14 eram mulheres, todos sem uso de medicação e 29 controles (12 mulheres). Uma versão emocional da tarefa stroop foi aplicada com estímulos verbais negativos, positivos e neutros apresentados por $200 \mathrm{~ms}$. O estudo também teve uma avaliação de valência emocional das palavras e de excitação provocada por elas. O escore de interferência da tarefa seguiu o mesmo procedimento já mencionado no estudo anterior. Os sintomas do TOC foram avaliados por meio do Y-BOCS, nenhum outro questionário de avaliação da patologia foi mencionado no estudo (Fan et al., 2014). 
O TR de pacientes com TOC foi maior e maiores amplitudes $\mathrm{P} 2$ em regiões frontais e centrais foram encontradas em relação a controles. Amplitudes de P3 também foram maiores no grupo TOC, sendo este aumento em média provocado por palavras negativas quando comparadas a neutras (Fan et al., 2014). O padrão inespecífico dos resultados levou os autores a concluírem que há um déficit geral na inibição da atenção, e não um viés a uma condição de estímulo irrelevante a tarefa em pacientes com TOC (análises de P3 e stroop emocional). Outra característica que complementa esta hipótese foi a indicação de processamento semântico das palavras quando o paradigma stroop pede para ignorá-las, isto poderia causar TR mais longos e a alocação da atenção para o distrator (Fan et al., 2014).

\section{DISCUSSÃO}

O objetivo do presente estudo foi descrever os achados de viés atencional voltado à ameaça em indivíduos com TOC, avaliados por medidas neuroelétricas e de neuroimagem. Quatro estudos satisfizeram os critérios determinados nesta revisão; e características metodológicas, resultados e conclusões foram considerados.

O hemisfério esquerdo apresentou mais áreas com alterações na ativação em pacientes com TOC nos estudos apresentados (Ischebeck et al., 2011; van den Heuvel et al., 2005). Considerando a suposta eficácia dos paradigmas utilizados para avaliar a atenção, essa informação diverge dos resultados de estudos em indivíduos saudáveis, que apontam para uma maior participação do hemisfério direito em tarefas que exigem o controle da atenção (Bench et al., 1993; Heilman \& Abell, 1980; Pardo et al., 1991). As áreas corticais citadas nos estudos com EEG são correspondentes aquelas com aumento na ativação observadas na RMF (Fan et al., 2014; Ischebeck et al., 2011; Thomsas et al., 2013; van den Heuvel et al., 2005).

$\mathrm{O}$ aumento da ativação de regiões fronto-estriatais encontrado por van den Heuvel et al. (2005) parece ser característico em pacientes com TOC, como vimos no modelo já citado (Abe et al., 2015; Ball et al. 2014). Nesse mesmo estudo, comparado a pacientes com hipocondria, o grupo com TOC apresentou aumento da ativação da área motora suplementar (AMS). Esta hiperativação apareceu em pacientes com TOC com relação a controles saudáveis, durante uma tarefa elaborada para ativar processos de controle inibitório (Yücel et al., 2007), e pode ter papel importante na manutenção do TOC. Estudos com estimulação magnética transcraniana associaram a inibição da AMS a diminuição dos sintomas no TOC (Kumar \& Chadda, 2011; Mantovani et al., 2013). O aumento da ativação destas áreas per se, não evidenciam o viés atencional voltado à ameaça no TOC. A AMS está associada aos movimentos distais e proximais dos membros, tarefas motoras simples, organização temporal dos movimentos, principalmente durante movimentos sequenciais (Tanji, 1994).

Observamos que as áreas citadas acima estão geralmente alteradas em pacientes com TOC (Ball et al., 2014) e per se não evidenciam o viés de atenção. Entretanto, uma avaliação da tarefa stroop emocional, durante RMF, em pessoas saudáveis demonstrou um aumento da atividade do córtex dorsolateral frontal relacionado à apresentação de palavras negativas e condições incongruentes (Compton et al., 2003). Este resultado reflete o sistema comum de manutenção da atenção na presença de distratores (Compton et al., 2003), o que não foi especificado como resultado no estudo de van den Heuvel et al. (2005), tanto para pacientes com TOC quanto para controles, podendo indicar uma facilidade de desvio de atenção que seria esperado para pacientes com TOC, mas não em controles.

No mesmo estudo, condições incongruentes aumentaram a ativação no córtex parietal esquerdo e diminuíram a ativação do giro parahipocampal. No caso de palavras negativas houve decréscimo na atividade da amígdala em indivíduos saudáveis (Compton et al., 2003). No estudo de van den Heuvel et al. (2005) ocorreu o oposto em pacientes com TOC, como maior ativação do giro parahipocampal esquerdo e tronco cerebral esquerdo durante condições incongruentes e maior ativação da amígdala durante palavras relacionadas ao TOC. Estas divergências de resultados, durante a mesma tarefa entre pacientes com TOC e indivíduos saudáveis reforçam que possa existir uma diferença no sistema da atenção em pacientes com TOC, com influência da amígdala e do hipocampo, podendo refletir em um viés atencional à ameaça. Complementarmente, o aumento da atividade do tronco cerebral e da amígdala durante condições incongruentes pode significar um estado de alerta a ameaça nestas condições.

O giro parahipocampal está relacionado à aprendizagem e evocação de informações espaciais (Aguirre et al., 1996; Maguire et al., 1996). A amígdala processa estímulos sensoriais, integra informações ambientais e promove a atenção apropriada e resposta motora (Bzdok et al., 2013). A excitação e a valência emocional são informações processadas separadamente pela amígdala. $O$ processamento da excitação pode contribuir para a regulação da atenção e modulação da memória (Gallagher \& Chiba, 1996). Houve ainda uma maior ativação do precuneus direito em pacientes com TOC (van den Heuvel et al., 2005), esta região está relacionada a auto-consciência, representações mentais durante o repouso e estratégias de imaginação mental centradas no indivíduo (Cavanna \& Trimble, 2006). O precuneus pode processar imagens visuoespaciais, memórias episódicas e evocá-las, bem como está relacionado à auto-consciência, representações mentais relacionadas ao próprio indivíduo durante o seu repouso e 
estratégias de imaginação mental centradas no indivíduo (Cavanna \& Trimble, 2006).

Nos estudos que utilizaram o EEG, o pico de P3 apresentou alterações como: maior amplitude durante condições negativas e neutras do teste stroop emocional, especificamente em regiões centrais, frontais e parietais do hemisfério esquerdo (Ischebeck et al., 2011); ou maiores somente em condições negativas, quando comparadas a neutras (Fan et al., 2014) em pacientes com TOC. Em um estudo com 71 pacientes com TOC, não medicados, estes apresentaram aumento de $\mathrm{P} 3$ no córtex orbitofrontal esquerdo, pré-frontal esquerdo, área parietal e temporal durante a tarefa auditory oddball comparados a controles, refletindo a hiperativação destas áreas, já relatada na possível fisiopatologia do TOC (Andreou et al., 2013). Entretanto, em pacientes pediátricos com TOC, amplitudes $\mathrm{P} 3$ foram menores nas posições central, frontal e temporal (Yamanuro et al., 2015).

Além disso, em um estudo de Di Russo et al. (2000), a atividade de $\mathrm{P} 3$ foi a mesma nas duas condições da tarefa go/no-go, em 8 pacientes com TOC sem TP e depressão comorbidas, mas quando comparados aos controles, P3 foi maior durante estímulos alvo e não houve diferenças para estímulos não-alvo (Di Russo et al., 2000). Houve diferenças topográficas de $\mathrm{P} 3$ estando centralizada em áreas frontais e centrais, durante estímulos alvo em pacientes, e no vértex em controles durante a mesma condição (Di Russo et al., 2000). Apesar das divergências, tais achados indicam um perfil de ativação cerebral diferente, entre pacientes com TOC e indivíduos saudáveis, em condições incongruentes e no mínimo desconfortáveis do ponto de vista emocional. Esse mesmo paradigma foi aplicado em dois grupos de pacientes com TOC, de início precoce e início tardio $(n=$ 59), em ambos os grupos houve um aumento da atividade $\mathrm{P} 3$, porém, menor que nos controles de acordo com o aumento da probabilidade da opção go. Este resultado foi atribuído a um déficit no controle da atenção (Keskin-Ergen et al., 2013).

Outros picos foram abordados nos estudos analisados, como: P1, P2 e N1. O viés atencional e níveis de ansiedade foram correlacionados ao aumento da amplitude P1. Li et al. (2005) também verificaram que em indivíduos com alto nível de ansiedade ocorreu um aumento da amplitude P1 na região occiptoparietal durante a substituição de imagens ameaçadores pelos estímulos alvo na tarefa cue target (Li et al., 2005). Em outros estudos, indivíduos ansiosos apresentaram maior amplitude P1 independente do tipo de estímulo (Rossignol et al., 2013), e o alto nível de ansiedade provocou maiores amplitudes em P2 durante estímulos negativos (Bar-Haim et al., 2005). Nesta revisão, amplitudes de P1 foram maiores durante condições de ameaça comparadas a neutras, com redução desta na posição fronto-parietal quando comparada à central. Em sequência, maiores amplitudes de P2 foram observadas acima da região fronto-parietal em pacientes com TOC comparados a controles nos resultados de Thomas et al. (2013). A semelhança com respeito ao aumento de amplitude dos dois picos entre indivíduos ansiosos e com TOC reforça a hipótese de que existe diferenças no sistema de atenção nestes indivíduos.

A ativação de áreas frontais, parietais e centrais durante estímulos ameaçadores representada pelo aumento da amplitude das ondas citadas (Fan et al., 2014; Ischebeck et al., 2011; Thomas et al., 2013), pode ser resultado da aplicação inadequada de recursos de atenção, possivelmente refletindo em um estado de alerta (regiões frontais e parietais), dificuldade de desviar a atenção da ameaça (córtex pré-frontal, ACC e OFC) e controle executivo (região fronto-parietal, córtices pré-frontal e frontal) (Cisler \& Koster, 2010; Petersen \& Posner, 2012). Contudo, se considerarmos que o nível de distração provocado pela ameaça é inversamente proporcional a ativação do córtex pré-frontal, os pacientes dos estudos analisados nesta revisão teriam se distraído menos com a ameaça contradizendo os demais resultados da ativação de outras áreas e da tarefa stroop emocional.

$\mathrm{O}$ aumento da latência do pico N1 para estímulos repetitivos foi correlacionado aos sintomas do TOC (Thomas et al., 2016) e aos níveis de ansiedade em indivíduos ansiosos (Bar-Haim et al., 2005). No estudo de O'Toole e Dennis (2012), os picos de ERP P1, P2 e P3 foram analisados em 49 indivíduos adultos não-ansiosos submetidos a um treinamento de atenção com foco e desvio, por meio do paradigma da tarefa dot probe. Os resultados foram o decréscimo da amplitude P1 do pré para o pós treino com desvio da atenção, para estímulos não ameaçadores e em todos os estímulos apresentados durante $100 \mathrm{~ms}$ do pós treino. Amplitudes de $\mathrm{P} 2$ e $\mathrm{P} 3$ aumentaram do pré para o pós treino e durante estímulos com $100 \mathrm{~ms}$ e não ameaçadores (somente P3). Os autores interpretaram estes resultados como indício de ausência de viés atencional (O’Toole \& Dennis, 2012). No pós treino maiores amplitudes $P 2$ foram associadas a viés atencional e vigilância na comparação entre estímulos ameaçadores e não ameaçadores (O’Toole \& Dennis, 2012). Porém, amplitude P2 foi menor para estímulos alvo da tarefa go/no-go em pacientes com TOC de checagem quando comparados a controles saudáveis e pacientes com TP (Thomas et al., 2016). Os três estudos de EEG desta revisão apresentaram resultados de aumento das amplitudes de P1, P2 e P3 em pacientes com TOC, especialmente, durante estímulos ameaçadores, podendo refletir, ao menos, uma ativação cerebral diferenciada durante o processamento de estímulos ameaçadores.

Considerando-se as limitações metodológicas dos estudos descritos, as limitações dos testes neuropsicológicos utilizados que não conseguem isolar efeitos exclusivos da atenção e o fato que não existe uma especificidade localizatória no sistema de atenção, é prematuro afirmar que os achados apontam para uma alteração específica de atenção no TOC. 


\section{CONCLUSÃO}

O conjunto de resultados encontrados não foram claros quanto a existência de viés atencional voltado à ameaça, mas cada estudo encontrou uma alteração na atenção em pacientes com TOC. No estudo de van den Heuvel et al. (2005), apesar de não ter sido identificado viés atencional voltado à ameaça por meio da tarefa stroop emocional, observou-se uma atenção para estímulos irrelevantes e ativação de áreas cerebrais específicas de acordo com as condições da tarefa. Estes pacientes supostamente apresentam uma resposta orientada aumentada a estímulos inesperados (Ischebeck et al., 2011), talvez por empregar uma atenção maior ao ambiente a fim de controlar situações inesperadas mantendo-se hipervigilante (Choi \& Lee, 2015), porém, não estritamente ameaçadoras.

Thomas et al. (2013) apresentou evidências de viés atencional voltado à ameaça por meio da tarefa stroop emocional e captação de EEG, e afirmou que pacientes com TOC possuem viés atencional precoce voltado à ameaça refletido pelo aumento da amplitude de P1 e P3 e latência de N1 durante estímulos ameaçadores. Aumento da amplitude $\mathrm{P} 3$ para valência emocional negativa em pacientes foi observada em Fan et al. (2014), porém os autores não atribuíram os resultados a um viés atencional voltado à ameaça, mas a uma disfunção na inibição da atenção para informações irrelevantes à tarefa. As considerações de Fan et al. (2014) condizem com as conclusões de Ischebeck et al. (2011) e van den Heuvel et al. (2005), nas quais a valência emocional do estímulo distrator é insignificante.

Devido as divergências e a pouca quantidade de estudos encontrados, parece mais coerente uma hipótese mais abrangente de uma alteração significativa na atenção em pacientes com TOC, possivelmente aumentando sua percepção a estímulos salientes em geral irrelevantes à tarefa corrente, podendo ou não prejudicá-la diretamente.

Os estudos de viés atencional voltado à ameaça no TOC com evidências neurobiológicas são poucos, apesar da recente associação deste com sua fisiopatologia. Entretanto, foram observadas, por meio desta revisão, peculiaridades da atenção em pacientes com TOC que podem contribuir para nortear futuros estudos. São necessárias investigações utilizando recursos de avaliação neurobiológica e não somente cognitiva. Amostras maiores e livres de medicação talvez possam contribuir significativamente para precisão dos resultados. A utilização de tarefas diversificadas para avaliação da atenção, realizadas em momentos distintos, pode ampliar os resultados possíveis.

\section{REFERÊNCIAS}

Abe, Y., Sakai, Y., Nishida, S., Nakamae, T., Yamada, K., Fukui, K., \& Narumoto, J. (2015). Hyper-influence of the orbitofrontal cortex over the ventral striatum in obsessive-compulsive disorder. European Neuropsychopharmacology: The Journal of the European College of Neuropsychopharmacology, 25(11), 1898-1905. http://doi.org/10.1016/j.euroneuro.2015.08.017

Aguirre, G. K., Detre, J. A., Alsop, D. C., \& D’Esposito, M. (1996). The parahippocampus subserves topographical learning in man. Cerebral Cortex, 6(6), 823-829. http://doi.org/10.1093/ cercor/6.6.823

American Psychiatric Association (1994). Diagnostic and statistical manual of mental disorders ( $4^{\mathrm{a}} \mathrm{ed}$.). DC: Author.

American Psychiatric Association (2013). Diagnostic and statistical manual of mental disorders ( $5^{\mathrm{a}} \mathrm{ed}$.). DC: Author.

Andreou, C., Leicht, G., Popescu, V., Pogarell, O., Mavrogiorgou, P., Rujescu, D., Giegling, I., Zaudig, M., Juckel, G., Hegerl, U., \& Mulert, C. (2013). P300 in obsessive-compulsive disorder: source localization and the effects of treatment. Journal of Psychiatric Research, 47(12), 1975-1983. http:// doi.org/10.1016/j.jpsychires.2013.09.003

Ball, T. M., Stein, M. B., \& Paulus, M. P. (2014). Toward the application of functional neuroimaging to individualized treatment for anxiety and depression. Depression and Anxiety, 31(11), 920-933. http://doi.org/10.1002/da.22299

Bar-Haim, Y., Lamy, D., \& Glickman, S. (2005). Attentional bias in anxiety: a behavioral and ERP study. Brain and Cognition, 59(1), 11-22. http://doi.org/10.1016/j.bandc.2005.03.005

Bar-Haim, Y., Lamy, D., Pergamin, L., Bakermans-Kranenburg, M. J., \& van IJzendoorn, M. H. (2007). Threat-related attentional bias in anxious and nonanxious individuals: a meta-analytic study. Psychological Bulletin, 133(1), 1-24. http://doi. org/10.1037/0033-2909.133.1.1
Beard, C. (2011). Cognitive bias modification for anxiety: current evidence and future directions. Expert Review of Neurotherapeutics, 11(2), 299-311. http://doi.org/10.1586/ ern.10.194

Beck, A. T., Ward, C. H., Mendelson, M., Mock, J., \& Erbaugh, J. (1961). An inventory for measuring depression. Archives of General Psychiatry, 4, 561-571.

Bench, C. J., Frith, C. D., Grasby, P. M., Friston, K. J., Paulesu, E., Frackowiak, R. S., \& Dolan, R. J. (1993). Investigations of the functional anatomy of attention using the Stroop test. Neuropsychologia, 31(9), 907-922. https:// doi.org/10.1016/0028-3932(93)90147-R

Bishop, S., Duncan, J., Brett, M., \& Lawrence, A. D. (2004). Prefrontal cortical function and anxiety: Controlling attention to threat-related stimuli. Nature Neuroscience, 7(2), 184-188. https://doi.org/10.1038/nn1173

Bzdok, D., Laird, A. R., Zilles, K., Fox, P. T., \& Eickhoff, S. B. (2013). An investigation of the structural, connectional, and functional subspecialization in the human amygdala. Human Brain Mapping, 34(12), 3247-3266. http://doi.org/10.1002/ hbm. 22138

Cavanna, A. E., \& Trimble, M. R. (2006). The precuneus: a review of its functional anatomy and behavioural correlates. Brain, 129, 564-583. . http://doi:10.1093/brain/awl004

Choi, S. A., \& Lee, J.-H. (2015). The effect of enhanced responsibility on attentional bias in obsessive-compulsive checkers. Journal of Obsessive-Compulsive and Related Disorders, 6, 27-32. http://doi.org/10.1016/j.jocrd.2015.04.003

Cisler, J. M., \& Koster, E. H. W. (2010). Mechanisms of attentional biases towards threat in the anxiety disorders: An integrative review. Clinical Psychology Review, 30(2), 203. http://doi. org/10.1016/j.cpr.2009.11.003 
Coles, M. E., \& Heimberg, R. G. (2002). Memory biases in the anxiety disorders: current status. Clinical Psychology Review, 22(4), 587-627.

Coles, M. E., Turk, C. L., \& Heimberg, R. G. (2007). Memory bias for threat in generalized anxiety disorder: the potential importance of stimulus relevance. Cognitive Behaviour Therapy, 36(2), 65-73. http://doi.org/10.1080/16506070601070459

Compton, R. J., Banich, M. T., Mohanty, A., Milham, M. P., Herrington, J., Miller, G. A., Scalf, P.E., Webb, A., \& Heller, W. (2003). Paying attention to emotion: An fMRI investigation of cognitive and emotional stroop tasks. Cognitive, Affective \& Behavioral Neuroscience, 3(2), 81-96.

Derogatis, L. R., \& Melisaratos, N. (1983). The brief symptom inventory: An introductory report. Psychological Medicine, 13(3), 595-605.

Di Russo, F., Zaccara, G., Ragazzoni, A., \& Pallanti, S. (2000). Abnormal visual event-related potentials in obsessivecompulsive disorder without panic disorder or depression comorbidity. Journal of Psychiatric Research, 34(1), 75-82.

Etkin, A., Egner, T., \& Kalisch, R. (2011). Emotional processing in anterior cingulate and medial prefrontal cortex. Trends in Cognitive Sciences, 15(2), 85-93. https://doi.org/10.1016/j. tics.2010.11.004

Fan, J., Zhong, M., Zhu, X., Lei, H., Dong, J., Zhou, C., \& Liu, W. (2014). An attentional inhibitory deficit for irrelevant information in obsessive-compulsive disorder: Evidence from ERPs. International Journal of Psychophysiology, 94(3), 420-426. http://doi.org/10.1016/j.ijpsycho.2014.11.002

Foa, E. B., Kozak, M. J., Salkovskis, P. M., Coles, M. E., \& Amir, N. (1998). The validation of a new obsessive-compulsive disorder scale: The Obsessive-Compulsive Inventory. Psychological Assessment, 10(3), 206-214. http://doi.org/10.1037/10403590.10.3.206

Friedman, D., Cycowicz, Y. M., \& Gaeta, H. (2001). The novelty P3: An event-related brain potential (ERP) sign of the brain's evaluation of novelty. Neuroscience and Biobehavioral Reviews, 25(4), 355-373.

Gallagher, M., \& Chiba, A. A. (1996). The amygdala and emotion. Current Opinion in Neurobiology, 6(2), 221-227.

Goodman, W. K., Price, L. H., Rasmussen, S. A., Mazure, C., Fleischmann, R. L., Hill, C. L., Heninger, G. R., \& Charney, D. S. (1989). The Yale-Brown Obsessive Compulsive Scale. I. Development, use, and reliability. Archives of General Psychiatry, 46(11), 1006-1011.

Harkness, E. L., Harris, L. M., Jones, M. K., \& Vaccaro, L. (2009). No evidence of attentional bias in obsessive compulsive checking on the dot probe paradigm. Behaviour Research and Therapy, 47(5), 437-443. http://doi.org/10.1016/j.brat.2009.02.004

Hartley, C. A., \& Phelps, E. A. (2010). Changing fear: the neurocircuitry of emotion regulation. Neuropsychopharmacology: Official Publication of the American College of Neuropsychopharmacology, 35(1), 136-146. https://doi.org/10.1038/npp.2009.121

Heilman, K. M., \& Van Den Abell, T. (1980). Right hemisphere dominance for attention: the mechanism underlying hemispheric asymmetries of inattention (neglect). Neurology, 30(3), 327-330.

Ischebeck, M., Endrass, T., Simon, D., \& Kathmann, N. (2011). Auditory novelty processing is enhanced in obsessivecompulsive disorder. Depression and Anxiety, 28(10), 915-923. http://doi.org/10.1002/da.20886

Jensen, A. R., \& Rohwer, W. D. (1966). The Stroop color-word test: a review. Acta Psychologica, 25(1), 36-93.

Keskin-Ergen, Y., Tükel, R., Aslantaş-Ertekin, B., Ertekin, E., Oflaz, S., \& Devrim-Üçok, M. (2013). N2 and P3 potentials in early-onset and late-onset patients with obsessive-compulsive disorder. Depression and Anxiety, 31(12), 997-1006. http://doi. org/10.1002/da.22212
Key, A. P. F., Dove, G. O., \& Maguire, M. J. (2005). Linking brainwaves to the brain: an ERP primer. Developmental Neuropsychology, 27(2), 183-215. http://doi.org/10.1207/ s15326942dn2702 1

Kondo, H., Osaka, N., \& Osaka, M. (2004). Cooperation of the anterior cingulate cortex and dorsolateral prefrontal cortex for attention shifting. NeuroImage, 23(2), 670-679. https:// doi.org/10.1016/j.neuroimage.2004.06.014

Kumar, N., \& Chadda, R. K. (2011). Augmentation effect of repetitive transcranial magnetic stimulation over the supplementary motor cortex in treatment refractory patients with obsessive compulsive disorder. Indian Journal of Psychiatry, 53(4), 340-342. http://doi.org/10.4103/0019-5545.91909

Kwon, J. S., Jang, J. H., Choi, J.-S., \& Kang, D.-H. (2009). Neuroimaging in obsessive-compulsive disorder. Expert Review of Neurotherapeutics, 9(2), 255-269. http://doi. org/10.1586/14737175.9.2.255

Kyrios, M., \& Iob, M. A. (1998). Automatic and strategic processing in obsessive-compulsive disorder: attentional bias, cognitive avoidance or more complex phenomena? Journal of Anxiety Disorders, 12(4), 271-292.

Lavy, E., van Oppen, P., \& van den Hout, M. (1994). Selective processing of emotional information in obsessive compulsive disorder. Behaviour Research and Therapy, 32(2), 243-246.

Li, X., Li, X., \& Luo, Y.-J. (2005). Anxiety and attentional bias for threat: an event-related potential study. Neuroreport, 16(13), 1501-1505.

Lima, R. F. (2005). Compreendendo os Mecanismos Atencionais [Understanding the attentional mechanisms]. Ciênc. cogn, $113-122$

Maguire, E. A., Frackowiak, R. S., \& Frith, C. D. (1996). Learning to find your way: a role for the human hippocampal formation. Proceedings. Biological Sciences / The Royal Society, 263(1377), 1745-1750. http://doi.org/10.1098/rspb.1996.0255

Mantovani, A., Rossi, S., Bassi, B. D., Simpson, H. B., Fallon, B. A., \& Lisanby, S. H. (2013). Modulation of motor cortex excitability in obsessive-compulsive disorder: an exploratory study on the relations of neurophysiology measures with clinical outcome. Psychiatry Research, 210(3), 1026-1032. http://doi.org/10.1016/j.psychres.2013.08.054

Mathews, A., Mackintosh, B., \& Fulcher, E. P. (1997). Cognitive biases in anxiety and attention to threat. Trends in Cognitive Sciences, 1(9), 340-345. http://doi.org/10.1016/S13646613(97)01092-9

Montgomery, S. A., \& Asberg, M. (1979). A new depression scale designed to be sensitive to change. The British Journal of Psychiatry: The Journal of Mental Science, 134, 382-389.

Moritz, S., Fischer, B.-K., Hottenrott, B., Kellner, M., Fricke, S., Randjbar, S., \& Jelinek, L. (2008). Words may not be enough! No increased emotional Stroop effect in obsessive-compulsive disorder. Behaviour Research and Therapy, 46(9), 1101-1104. http://doi.org/10.1016/j.brat.2008.05.005

Muller, J., \& Roberts, J. E. (2005). Memory and attention in Obsessive-Compulsive Disorder: a review. Journal of Anxiety Disorders, 19(1), 1-28. http://doi.org/10.1016/j. janxdis.2003.12.001

Nakao, T., Okada, K., \& Kanba, S. (2014). Neurobiological model of obsessive-compulsive disorder: Evidence from recent neuropsychological and neuroimaging findings. Psychiatry and Clinical Neurosciences, 68(8), 587-605. http://doi.org/10.1111/ pcn. 12195

O'Toole, L., \& Dennis, T. A. (2012). Attention training and the threat bias: an ERP study. Brain and Cognition, 78(1), 63-73. http://doi.org/10.1016/j.bandc.2011.10.007

Pardo, J. V., Fox, P. T., \& Raichle, M. E. (1991). Localization of a human system for sustained attention by positron emission tomography. Nature, 349(6304), 61-64. https://doi. org/10.1038/349061a0 
Petersen, S. E., \& Posner, M. I. (2012). The attention system of the human brain: 20 years after. Annual Review of Neuroscience, 35 , 73-89. http://doi.org/10.1146/annurev-neuro-062111-150525

Pilowsky, I. (1967). Dimensions of hypochondriasis. The British Journal of Psychiatry: The Journal of Mental Science, 113(494), 89-93.

Posner, M. I. (1992). Attention as a Cognitive and Neural System. Current Directions in Psychological Science, 1(1), 11-14.

Posner, M. I. (1994). Attention: the mechanisms of consciousness. Proceedings of the National Academy of Sciences of the United States of America, 91(16), 7398-7403.

Rao, N. P., Arasappa, R., Reddy, N. N., Venkatasubramanian, G., \& Reddy, Y. C. J. (2010). Emotional interference in obsessivecompulsive disorder: a neuropsychological study using optimized emotional Stroop test. Psychiatry Research, 180(23), 99-104. http://doi.org/10.1016/j.psychres.2009.10.017

Rauch, S. L. (2003). Neuroimaging and neurocircuitry models pertaining to the neurosurgical treatment of psychiatric disorders. Neurosurgery Clinics of North America, 14(2), 213-223, vii-viii.

Rossignol, M., Campanella, S., Bissot, C., \& Philippot, P. (2013). Fear of negative evaluation and attentional bias for facial expressions: an event-related study. Brain and Cognition, 82(3), 344-352. http://doi.org/10.1016/j.bandc.2013.05.008

Sanavio, E. (1988). Obsessions and compulsions: The Padua inventory. Behaviour Research and Therapy, 26(2), 169-177. http://doi.org/10.1016/0005-7967(88)90116-7

Spielberger, C. D., Gorsuch, R. L., Lushene, R., Vagg, P. R., \& Jacobs, G. A. (1983). Manual for the State-Trait Anxiety Inventory. CA: Consulting Psychologists Press.

Tanji, J. (1994). The supplementary motor area in the cerebral cortex. Neuroscience Research, 19(3), 251-268. http://doi. org/10.1016/0168-0102(94)90038-8

Thomas, S. J., Gonsalvez, C. J., \& Johnstone, S. J. (2013). Neural time course of threat-related attentional bias and interference in panic and obsessive-compulsive disorders. Biological Psychology, 94(1), 116-129. http://doi.org/10.1016/j. biopsycho.2013.05.012

Thomas, S. J., Gonsalvez, C. J., Johnstone, S.J. (2016). Electrophysiology of facilitation priming in obsessivecompulsive and panic disorders. Clinical Neurophysiology, 127(1), 464-478. doi: 10.1016/j.clinph.2015.05.026.

van den Heuvel, O. A., Veltman, D. J., Groenewegen, H. J., Witter, M. P., Merkelbach, J., Cath, D. C., Balkom, A. J. L. M., van Oppen, P., \& van Dyck, R. (2005). Disorder-specific neuroanatomical correlates of attentional bias in obsessivecompulsive disorder, panic disorder, and hypochondriasis. Archives of General Psychiatry, 62(8), 922-933. http://doi. org/10.1001/archpsyc.62.8.922

Vogel, E. K., \& Luck, S. J. (2000). The visual N1 component as an index of a discrimination process. Psychophysiology, 37(2), 190-203.

Wu, D., Deng, H., Xiao, X., Zuo, Y., Sun, J., \& Wang, Z. (2017). Persistent Neuronal Activity in Anterior Cingulate Cortex Correlates with Sustained Attention in Rats Regardless of Sensory Modality. Scientific Reports, 7, 43101. https://doi. org/10.1038/srep43101

Yamamuro, K., Ota, T., Nakanishi, Y., Matsuura, H., Okazaki, K., Kishimoto, N., Takahashi, H., Iwasaka, H., Iida, J., \& Kishimoto, T. (2015). Event-related potentials in drug-naïve pediatric patients with obsessive-compulsive disorder. Psychiatry Research. http://doi.org/10.1016/j. psychres.2015.09.02

Yücel, M., Harrison, B. J., Wood, S. J., Fornito, A., Wellard, R. M., Pujol, J., Clarke, K., Phillips, M. L., Kyrios, M., Velakoulis, D., \& Pantelis, C. (2007). Functional and biochemical alterations of the medial frontal cortex in obsessive-compulsive disorder. Archives of General Psychiatry, 64(8), 946-955. http://doi. org/10.1001/archpsyc.64.8.946 